\title{
Review of the Upside of Your Dark Side: Why Being Your Whole Self - Not Just Your "Good" Self - Drives Success and Fulfillment, by Todd Kashdan and Robert Biswas-Diener
}

\author{
Acacia Parks
}

\section{Overview}

The Upside of Your Dark Side is the result of collaboration between Todd Kashdan, a research powerhouse and vocal critic from within the positive psychology movement, and Robert Biswas-Diener, an accomplished author, coach, consultant, and researcher in his own right. The resulting product gives readers the best of both authors' strengths: outstanding empirical rigor, vibrant writing, and groundbreaking ideas. The central thesis of Upside is to turn on its head the common wisdom that negative emotions are bad and should be avoided; they show how in moderation, in the right situations, three key negative emotions (anger, guilt, and anxiety) can be tremendously beneficial. They also argue that happiness and mindfulness, two constructs often thought to be universally beneficial, are not all good. They close with the proposition that a person who avoids negativity misses out on the benefits of being "whole" - a person who experiences both positive and negative emotions and harnesses each to their full potential.

\section{Who is the Audience of Upside?}

Like Kashdan's previous book, Curious?, one of the greatest strengths of Upside is its appeal to a very broad audience - its contents are certainly worth reading for the self-help market (and is written very accessibly to make that possible), but researchers in positive psychology also need to hear what Kashdan and Biswas-Diener have to say. As a researcher myself, I am always impressed when a book manages to draw my attention to research that is relevant to my work but that I am unaware of. Even more impressive, there were several occasions when the authors showed me a new perspective to research that I have previously read and consumed on my own! I am proud to say that my research program has benefitted by reading this selfhelp book - that hasn't happened to me since Haidt's The Happiness Hypothesis almost 10 years ago. In short, from the casual reader interested in learning more about the science of emotion to the seasoned researcher looking for some new perspectives, Upside delivers something worthwhile.

\section{Strengths and Weaknesses}

Like any work, Upside has both strengths and weaknesses. I noted one strength above: its appeal to a very broad audience. I was also pleased to see that, in a sea of self-help books on happiness that are informational but do not provide any real, implementable suggestions for 
improvement, Upside provides concrete advice for using the scientific findings they describe to change one's behaviors. For example, the authors provide a "speed limit" metaphor for helping readers understand when anger is and isn't going to be helpful - basically, it has to be intense enough to get your attention (not just "irk" you) but not so intense that you lose logical thought or the ability to problem-solve. By helping readers apply this metaphor to figure out just how angry they are, and then advising readers to attempt problem-solving at just the optimal "speed" (or level of anger), they turn an otherwise abstract concept ("harness your anger") into something the reader may actually be equipped to try after finishing the chapter. The sections on Guilt and Anxiety also provide similarly excellent suggestions.

One problem I noted was in Chapter 4's discussion of the pitfalls of happiness. It is true that happiness, as a mood state, is not entirely positive (and they note several key disadvantages, such as thinking less critically), and I value the authors' description of that research, as I do think it is important for readers to be educated on the nuances of happiness that it isn't always optimal. However, in my reading, the case for the pitfalls of happiness (that, as they say, the pursuit of happiness "often backfires") is somewhat overstated. They spent a disproportionate amount of attention on work by Mauss and colleagues which finds that people who want to be happy suffer from a paradoxical and frustrating inability to actually seek it. The "paradoxical effect" observed by Mauss and colleagues is a relatively new phenomenon, and Mauss's work is by no stretch the only research that speaks to the topic, though the prose of Upside would suggest otherwise. Other work (including data in my own lab) finds that valuing happiness - particularly if it is assessed using a different measure than Mauss's - means better motivation and subsequently more successful efforts at selfimprovement.

On a more minor note, although I enjoyed Chapter 5, "Beyond the Obsession With Mindfulness" - it's one of the most playful in the book, and contains some interesting ideas - in my reading, it detracted from the unity of the book. The authors open by discussing how Western societies have become obsessed with creature comforts - a direct lead-in to the general hypothesis of the book, that negative emotions may be uncomfortable, but we need them. Encouraging mindlessness doesn't really fit into that general argument. If anything, people often adopt mindlessness to avoid negative feelings and struggle with mindfulness because it involves accepting unpleasant feelings. Indeed, the concluding chapter of the book comes full circle to endorse "wholeness" - which in many ways sounded a lot like what proponents of mindfulness strive for (they say, for example, that readers should "quit labeling ... inner states as 'good/bad' or 'positive/negative." If that's not mindfulness, I don't know what is!). In short, the mindfulness chapter felt like a digression from the main thread of the book, though that thread was promptly picked up again in Chapter 6. I found myself less convinced by the science, and subsequently less interested in following the chapter's recommendations - not due to any problems with the science itself or the scientific rigor of the chapter - but because the book's otherwise very compelling rationale and background argumentation did not squarely apply to Chapter 5's contents as it did to the others.

\section{Conclusions}

The Upside of Your Dark Side is an expertly written, funny, thoughtful, and irreverent book. For consumers of self-help and members of the general public, it provides many practical pieces of advice while also giving the reader a solid understanding of a wealth of interesting and personally relevant research. As an academic myself, however, I cannot finish this book review without also lauding Upside for its relevance as a piece of thought leadership in psychology. 
I said something similar about Kashdan's last book, Curious?, but it's true here as well: while the book clearly set out to distinguish itself from positive psychology, reading the final chapter, I couldn't help but feel like Upside is perhaps even more "positive psych" than the actual positive psychology movement. It's everything positive psychology is, but more: more interdisciplinary, more nuanced, more rigorous, and more self-reflective. This is showcased most of all in the final two chapters, in particular, (Ch. 6, "The Teddy Effect" and Ch. 7, "The Whole Enchilada") which masterfully weave together research from so many disparate areas of psychology - Kashdan's home turf of curiosity, theoretical work on the nature of happiness (an area to which both Biswas-Diener and Kashdan are vocal contributors), basic social psychology, decision-making, and industrial/organizational psychology. The interrelation between these fields and their relevance to happiness is illustrated using deftly executed examples from both history and popular culture. If you've ever had one of those "aha" moments where suddenly, you realize that everything is connected by one grand theme - that's how I felt as I closed the book. Happiness, and its pursuit, is a much broader topic than it gets credit for in the ongoing discourse of positive psychology; the wide-ranging, yet well-focused conversation started by Kashdan and Biswas-Diener in Upside is an important start in advancing academic thought on happiness.

\section{Authors}

Acacia Parks

Hiram College

parksac@hiram.edu

\section{Publishing Timeline}

Received 26 January 2015

Accepted 26 January 2015

Published 26 January 2015

\section{References}

Kashdan, T., \& Biswas-Diener, R. (2014). The Upside of Your Dark Side: Why Being Your Whole Self - Not Just Your "Good" Self - Drives Success and Fulfillment. New York, Hudson Street Press. 304 pp., \$25.95 hardcover + \$12.99 epub, ISBN: 9781594631733. 